$0,3-0,4 \mathrm{~g}$ des zu prüfenden Fettes, genau gewogen, löst man in $20 \mathrm{cc}$ Chloroform, fügt $20 c c-$ bei trocknenden Oelen $30 c c-$ Jodlösung ${ }^{1}$ ) hinzu und stellt 24 Stunden lang bei Seite. Ferner bringt man noch je $20 \mathrm{cc}$ in zwei Glasstöpselflaschen derselben Grösse ${ }^{2}$ ) und Beschaffenheit wie bei dem eigentlichen Versuch, setzt je $20 \mathrm{cc}$ Chloroform hinzu und titrirt die eine, nachdem man $20 c c$ Jodkaliumlösung (1:10) hinzugefügt hat, sofort, während man die andere Probe als blinden Versuch genau wie den eigentlichen Versuch behandelt und nach 24 Stunden unter Zusatz von $20 \mathrm{cc}$ Jodkaliumlösung und $200 \mathrm{cc}$ Wasser mit ZehntelThiosulfat den Jodüberschuss zurückmisst. Aus den erhaltenen Werthen berechnet man die Jodzahl in bekannter Weise.

Bei Besprechung der Methoden von Welmans ${ }^{3}$ ) und von Gantter ${ }^{4}$ ) bestätigt Dieterich die grössere Haltbarkeit der von diesen beiden Verfassern angegebenen Jodlösungen, findet jedoch die mit diesen Lösungen erhaltenen Werthe weniger übereinstimmend als bei dem von ihm befolgten Hübl'schen Verfahren. Zudem sollen die neven Lösungen unangenehmer mit der Pipette anzusaugen sein als die $\mathrm{Hübl}$ 'schen Lösungen. Dieterich empfiehlt, Jod und Quecksilberchlorid in getrennten Lösungen vorräthig zu halten und nur so viel zur Hübl'schen Lösung zu mischen, als man innerhalb acht Tagen verbraucht.

Bezüglich der von Dieterich ${ }^{5}$ ) bestimmten Jodzahlen der Balsame, Harze und Gummiharze kann hier nur auf das Original Bezug genommen werden. Dasselbe gilt von den sehr ausführlichen Dieterich'schen ${ }^{6}$ ) Angaben über die Untersuchung der Balsame, Harze und Gummiharze überhaupt.

Bei Prüfung einer Anzahl Muster känflicher Oelsäure durch Titriren mit alkoholischer Kalilange fand Eugen Dieterich ${ }^{7}$ ), dass $178,73-194,13 \mathrm{mg}$ Kaliumhydroxyd zur Sättigung des Säuregehaltes erforderlich waren. Dies entspricht einem Gehalte von $90-97,28 \%$ Oelsäure. Hiernach könnte ein Gehalt von $90 \%$ Oelsäure im Acidum oleïnicum crudum als Mindestgehalt einer guten Waare angesehen werden. Die Jodzahl derselben Oelsäureproben schwankte von $74,20-85,87$.

1) von welcher $20 c c 30$ bis $36 c c$ Zehntel-Thiosulfatlösung entsprechen.

2) Inhalt 500 bis $700 c c$.

3) Vergl. diese Zeitschrift 32, 497.

4) Diese Zeitschrift 32, 178 น. 181.

5) Helfenberger Anmalen 1891, S. 23.

6) Ebendaselbst 1891, S. 22 und 1892, S. 14.

7) Helfenberger Amalen 1891, S. 10. 
Später ${ }^{1}$ ) fand Verfasser bei Bestimmung der Jodzahl selbst dargestellter reiner Oelsäure 89,80 und 90,05 . Theoretisch würde ein Molecuil Oelsäure 2 Atome Jod, 100 Theile Oelsäure also 90,07 Theile Jod aufnehmen. Die Bestimmung der Jodzahl dürfte hiernach eine Schätzung des Gehaltes an Oelsäure ermöglichen. Dieterich normirt das Minimum der Jodzahl für eine gute Handelswaare auf 178,73 entsprechend $90 \%$.

Identitätsreactionen verschiedener Extracte sind von Eugen Dieterich ${ }^{2}$ ) bearbeitet worden. Bei der leichten Zugänglichkeit des Originales für den beschränkten Kreis der Interessenten wird auf die ziemlich umfangreiche Arbeit hier am besten wohl nur aufmerksam gemacht. Dasselbe gilt von den neuesten Veröffentlichungen des Verfassers über die Untersuchung der Extracte. ${ }^{3}$ )

Eine Anleitung zur Prüfung und Gehaltsbestimmung der Arzneistoffe für Apotheker, Chemiker, Aerzte und Sanitätsbeamte haben Richard Pribram und Neumann Wender herausgegeben. ${ }^{4}$ ) Ich verfehle nicht, hier auf dieses Buch aufmerksam zu machen.

\section{Zur Untersuchung und Werthbestimmung von Kreosotpillen} haben Constantin Monheim ${ }^{5}$ ) und A. Schlicht ) Mittheilungen gemacht, welche im Anschluss an die ersten Angaben des letztgenannten Verfassers ${ }^{7}$ ) hier jedoch nur erwähnt werden können, da die bezüglichen Arbeiten noch nicht $\mathrm{zu}$ dem erforderlichen Abschluss gekommen sind.

Die Löslichkeit einiger Balsame und Harze in verschiedenen Lösungsmitteln, welche bei einschlägigen Untersuchungen sehr zu berücksichtigen ist, hat E. Dieterich ${ }^{8}$ ) festgestellt. Die Ergebnisse sind in der nachstehenden Tabelle Seite 630 u. 631 zusammengestellt. Dieterich macht noch besonders darauf aufmerksam, dass eine Verfälschung des Peru- und des Tolu-Balsams durch eine quantitative Bestimmung des in Petroläther Unlöslichen sehr wohl möglich sein dürfte.

1) Helfenberger Annalen 1892, S. 1.

2) Helfenberger Annalen 1891, S. 50.

3) Eibendaselbst 1892, S. 33.

4) Wien 1893, Moritz Perles.

5) Ber. d. Pharm. Gesellsch. zu Berlin 3, 99.

6) Pharm. Zeitung 38, 330.

7) Diese Zeitschrift 32, 375.

8) Helfenberger Annalen 1891, S. 30. 